\title{
Liver fibrosis during clinical ascertainment of glycogen storage disease type III: a need for improved and systematic monitoring
}

\author{
Carine A. Halaby, MD ${ }^{1}{ }^{1}$, Sarah P. Young, $\mathrm{PhD}^{1}$, Stephanie Austin, MS, MA ${ }^{1}$, Ela Stefanescu, BS ${ }^{1}$, \\ Deeksha Bali, MD, PhD ${ }^{1}$, Lani K. Clinton, MD, PhD², Brian Smith, MD, MPH ${ }^{3}$, \\ Surekha Pendyal, MSc, RD ${ }^{1}$, Jariya Upadia, MD ${ }^{1}$, Gary R. Schooler, MD ${ }^{4}$, \\ Alisha M. Mavis, $\mathrm{MD}^{5}$ and Priya S. Kishnani, MD ${ }^{1}{ }^{1}$
}

Purpose: In glycogen storage disease type III (GSD III), liver aminotransferases tend to normalize with age giving an impression that hepatic manifestations improve with age. However, despite dietary treatment, long-term liver complications emerge. We present a GSD III liver natural history study in children to better understand changes in hepatic parameters with age.

Methods: We reviewed clinical, biochemical, histological, and radiological data in pediatric patients with GSD III, and performed a literature review of GSD III hepatic findings.

Results: Twenty-six patients (median age 12.5 years, range 2-22) with GSD IIIa $(n=23)$ and IIIb $(n=3)$ were enrolled in the study. Six of seven pediatric patients showed severe fibrosis on liver biopsy (median [range] age: 1.25 [0.75-7] years). Markers of liver injury (aminotransferases), dysfunction (cholesterol, triglycerides), and glycogen storage (glucose tetrasaccharide, $\mathrm{Glc}_{4}$ ) were elevated at an early age, and decreased significantly thereafter $(p<0.001)$. Creatine phosphokinase was also elevated with no significant correlation with age $(p=0.4)$.

Conclusion: Liver fibrosis can occur at an early age, and may explain the decrease in aminotransferases and $\mathrm{Glc}_{4}$ with age. Our data outlines the need for systematic follow-up and specific biochemical and radiological tools to monitor the silent course of the liver disease process.

Genetics in Medicine (2019) 21:2686-2694; https://doi.org/10.1038/s41436019-0561-7

Keywords: hepatocellular fibrosis; cirrhosis; GSD III liver; urinary glucose tetrasaccharide

\section{INTRODUCTION}

Glycogen storage disease type III (GSD III; OMIM 232400) is an autosomal recessive disorder caused by a deficiency of glycogen debranching enzyme (GDE) encoded by the AGL gene. GDE deficiency causes an accumulation of limit dextrin-like molecules in the cytoplasm of hepatocytes, myocytes, and other tissues. There are two subtypes based on the tissues involved and location of the deficient enzyme. ${ }^{1,2}$ GSD IIIa accounts for $85 \%$ of cases and involves both muscle and liver, compared with liver-only findings in GSD IIIb. GSD IIIb is associated with the presence of either of two pathogenic variants in exon 3, c.18_19delGA (p. Gln6HisfsX20) or c.16C>T (p.Gln6X), combined with a second pathogenic variant that may occur at other locations. ${ }^{3}$ Multiple pathogenic variants that result in GSD IIIa have been reported throughout the 35 exons of the AGL gene. ${ }^{2,3}$
Individuals with GSD III typically present within the first few months of life ${ }^{1}$ with ketotic hypoglycemia, elevated aminotransferases, hyperlipidemia, hepatomegaly, and growth retardation. During adolescence, clinical "hepatic improvement" becomes more apparent with decreases in liver size and aminotransferases, while muscle disease becomes more pronounced. However, longterm hepatic complications have been reported, including liver fibrosis, cirrhosis, hepatocellular adenomas (HCA), and hepatocellular carcinoma (HCC). ${ }^{4,5}$ Mouse models and a naturally occurring canine model of GSD IIIa have shown similar trends in these liver parameters, with initial increases in aminotransferases and hepatomegaly, followed by a decrease with age. Liver biopsies from the animal models provided evidence of ongoing liver damage, ${ }^{6,7}$ suggesting the decrease in liver parameters with age are associated with a progression, and not an improvement, of

${ }^{1}$ Division of Medical Genetics, Department of Pediatrics, Duke University Medical Center, Durham, NC, USA; ${ }^{2}$ Department of Pathology, Duke University Medical Center, Durham, NC, USA; ${ }^{3}$ Division of Neonatal-Perinatal Medicine, Department of Pediatrics, Duke University Medical Center, Durham, NC, USA; ${ }^{4}$ Department of Radiology, Duke University Medical Center, Durham, NC, USA; ${ }^{5}$ Division of Gastroenterology, Hepatology and Nutrition, Department of Pediatrics, Duke University Medical Center, Durham, NC, USA. Correspondence: Priya S. Kishnani (priya.kishnani@duke.edu)

These authors contributed equally: Carine Adonis Halaby, Sarah P. Young

Submitted 12 November 2018; accepted: 21 May 2019

Published online: 2 July 2019 
the liver disease. This is further supported by similar trends of a urinary glucose tetrasaccharide biomarker of glycogen storage, ${ }^{8}$ $\mathrm{Glc}_{4}$, which is increased in GSD III. ${ }^{7}$ Current treatment of GSD III focuses on strict dietary control using cornstarch and a high protein diet, to maintain euglycemia. ${ }^{9}$ Dietary intervention can prevent hypoglycemia and improve growth, ${ }^{10}$ but its impact on liver disease progression is unknown. ${ }^{11,12}$ This highlights the need for natural history studies to better characterize hepatic disease in pediatric patients with GSD III. Case reports and patient cohort studies have described liver complications (Table S1), but have not addressed the issues of age at onset, severity in the younger GSD III population, and progression of liver injury.

The objective of this study was to describe the natural history of liver disease in GSD III in pediatric patients.

\section{MATERIALS AND METHODS}

This was a single-center, retrospective, longitudinal natural history study of liver manifestations in pediatric patients (aged $\leq 22$ years at the most recent assessment) with GSD III followed at Duke University Hospital. The study was approved by the Duke University Institutional Review Board (IRB). Informed consent or a decedent waiver was obtained from patients or a parent/guardian for those $<18$ years (IRB Pro00013699). Histological evaluations of liver biopsies were performed for pediatric patients where available. Additionally, evaluations were performed for a group of adult patients with GSD III $(n=5)$ to allow comparison with the pediatric population, with the aim of obtaining insight on liver disease progression.

\section{Data collection}

Data were collected for gender, age, ethnicity, diagnostic results, ${ }^{13}$ medical history, imaging studies, liver biopsies, and hepatic findings on physical examination. Information on cardiac involvement was previously reported. ${ }^{14}$ Height and body mass index percentiles were recorded using the WHO growth chart. Longitudinal laboratory values were recorded where available and included creatine phosphokinase (CPK), alanine aminotransferase (ALT), aspartate aminotransferase (AST), gamma glutamyl transferase (GGT), urinary $\mathrm{Glc}_{4}$, triglycerides, total cholesterol, high density and low density lipoproteins (HDL, LDL), platelet count, prothrombin time (PT), bilirubin, albumin, glucose, and 3hydroxybutyrate levels. Lipid profiles were routinely performed after 3-4 hours of fasting because of hypoglycemia risk in GSD III and were considered abnormal when elevated above the reference range. ${ }^{15} \mathrm{Glc}_{4}$ was measured as previously reported ${ }^{16}$ and compared with age-specific reference ranges (1-3 years: $<8.3$ [95th percentile, 95\% CI: 6.5-9.7, $n=77$ ]; $>3$ years: $<3 \mathrm{mmol} / \mathrm{mol}$ creatinine [CN], [95\% CI: 2.8-4.9, $n=328$ ]).

All patients were on dietary therapy under the management of a metabolic dietitian. Diet diaries included blood glucose and ketone levels checked using Precision Xtra ketone monitor and test strips at home for three days before clinic visits. Five pediatric patients and one adult patient also had weeklong continuous glucose monitoring (CGM). ${ }^{17}$

An experienced physical therapist assessed manual muscle testing (MMT) to evaluate muscle strength. Key lower extremity muscles were tested bilaterally, against the examiner's resistance, and included the following muscle groups: hip adductors, abductors, extensors and flexors, ankle dorsiflexors, and plantar flexors. Scores ranged from 0 (no muscle activation) to 5 (ability to contract with full range of motion, against gravity and with maximal resistance). ${ }^{18}$ Scores of $3+$ indicated an ability to move through full range antigravity, with resistance only through part of the range. Muscle actions $\leq 3+$ were defined as "weak." Data on ambulatory status and use of assistive devices was also recorded.

Imaging studies, including abdominal ultrasound (US), magnetic resonance imaging (MRI), or computerized axial tomography (CT) scans, were evaluated for liver size, presence of liver masses, and change in echotexture. Most recent studies were included, and those performed at Duke were reviewed by an experienced pediatric radiologist (G.S.).

Liver biopsies were reviewed by an expert hepatopathologist (L. K.C.). Biopsies were performed for diagnostic purposes, except for two pediatric patients (aged 7 and 20 years) and in all adult patients, who had biopsies performed because of a clinical concern for liver disease. The Batts-Ludwig system (also known as modified Scheuer system) was used to stage the extent of fibrosis. ${ }^{19}$ Although this system was originally developed to unify reporting for chronic hepatitis (viral and autoimmune), it is a highly reproducible staging system. There are no unified reporting systems for GSD, thus the Batts-Ludwig staging system was applied in this study as follows: stage 1: portal fibrosis; stage 2: periportal fibrosis where short, spike-like fibrous extensions are seen radiating from the portal tracts; stage 3: bridging fibrosis where the aforementioned short, spike-like fibrous extensions reach all the way to another portal tract; and stage 4: regenerative nodule formation (cirrhosis).

\section{Statistical analyses}

Continuous data was expressed as frequencies (\%), mean \pm standard deviation (SD), or median and range where the sample size was small. Categorical data was expressed as proportions. The relationships between continuous measures were examined using generalized estimating equations to account for multiple observations per patient. STATA 15.0 (College Station, TX) was used for the analysis.

\section{Literature review}

A literature search of the PubMed database was performed from 1967 to 2018 using the keywords "glycogenosis type III," "glycogen storage disease type III," "Cori disease," and "debrancher enzyme deficiency." All retrospective and prospective studies reported in English, including human and animal models, systematic reviews, and case studies that described any hepatic symptom or outcome were included.

\section{RESULTS}

\section{Demographic and baseline disease characteristics}

Between 1995 and 2017, 26 patients with GSD III met inclusion criteria with a median follow-up time of 10.5 years (1-21). Characteristics of the 26 patients are described in Table S2. Median age at diagnosis was 0.96 years (0.33-4.4). 
At the most recent assessment, the median age was 12.5 years $(2-22)$ and $13(50 \%)$ patients were $\leq 10$ years of age. Median (range) percentiles for body mass index (BMI) and height were 80 th \% (25th-99th) and 38th \% (1st-99th), respectively.

\section{AGL pathogenic gene variant analysis}

Twenty-four patients from 23 families had AGL gene analysis performed (Table S3). Pathogenic variants on both alleles were detected in 23 patients, a majority of whom (70\%) were compound heterozygotes. A single heterozygous pathogenic variant was found in one patient, and the diagnosis of GSD IIIa was confirmed by enzyme analysis. Twenty-seven unique pathogenic variants were identified, including three novel pathogenic variants (c.4259+1G >A, c.3235C > T, c.3299delG) not previously reported. One missense variant, c.3866T $>C$ (p.Leu1289Pro) was classified as a variant of unknown significance, and the diagnosis was confirmed by enzyme analysis. The distribution of pathogenic variant types included missense $(20 \%)$, nonsense $(28 \%)$, splice site $(24 \%)$, exon deletion (4\%), and small frameshift deletions and insertions (24\%). Twenty-two variants (79\%) were predicted to produce truncated proteins and seven patients (29\%) had truncating variants on both alleles. All three patients with GSD IIIb were compound heterozygotes for the IIIb c.18_19delGA pathogenic variant and one other pathogenic variant.

\section{Liver histology findings}

Thirteen liver histological analyses were reviewed by an expert hepatopathologist, including seven obtained from outside hospitals. Data included six pediatric patients from the study cohort (patient ID 4, 9, 28, 35, 36, 37 at ages 2.8 years, 15 months, 11 months, 7 years, 11 months, and 9 months, respectively), two additional pediatric patients (aged 2.2 and 20 years), and five adults (median age 33 years, range: $24-39$ ) (see Table S2). All 13 patients had histological changes consistent with liver fibrosis. In two patients, aged 11 months and 33 years, the biopsies showed periportal fibrosis (stage 2). The remaining 11 patients (median age 20 years, range 9 months-39 years) demonstrated advanced fibrosis. Among these 11 patients, 9 patients had bridging fibrosis (stage 3), including 7 in the pediatric cohort (ages 9 months, 11 months, 15 months, 2.2 years, 2.8 years, 7 years, and 20 years), and 2 in the adult cohort (ages 33 and 39 years). Two adult patients had cirrhosis (stage 4 ) at age 24 and 33 years.

The distribution of fibrosis was not always uniformly present throughout the biopsy sample. For example, although a biopsy from patient 37, aged 9 months, had areas of bridging fibrosis in some slides, there were also areas of histologically classic GSD characterized by cytoplasmic clearing and pyknotic nuclei in other slides (Fig. 1a, b) without any evidence of fibrosis on the Masson trichrome stain (Fig. 1c). These findings are in stark contrast to Fig. 2 (patient 35, 7 years), which shows an example of advanced fibrosis on both routine hematoxylin and eosin-stained slides (Fig. 2a) as well as on the Masson trichrome stain (Fig. 2b). Inflammation was present in $9 / 13$ patients (70\%), which was not correlated with the degree of fibrosis. The a

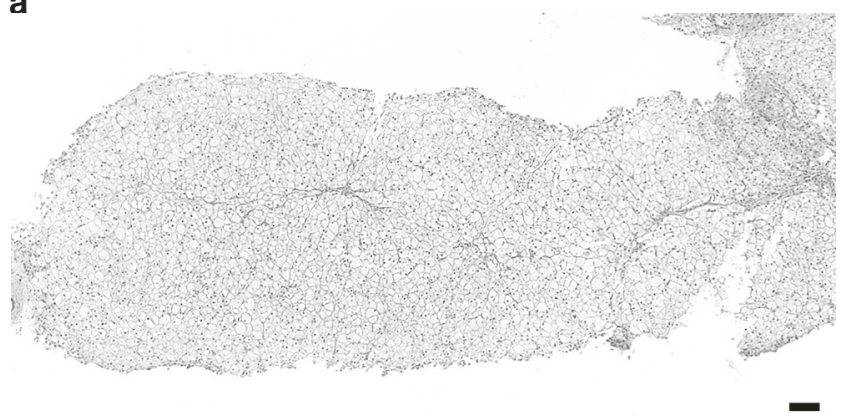

b

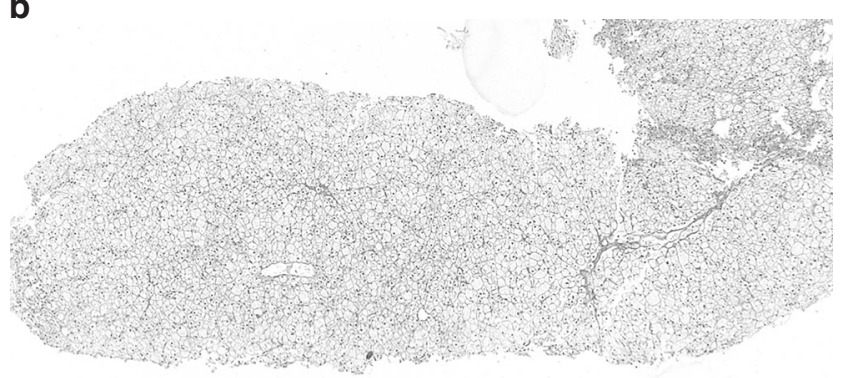

C

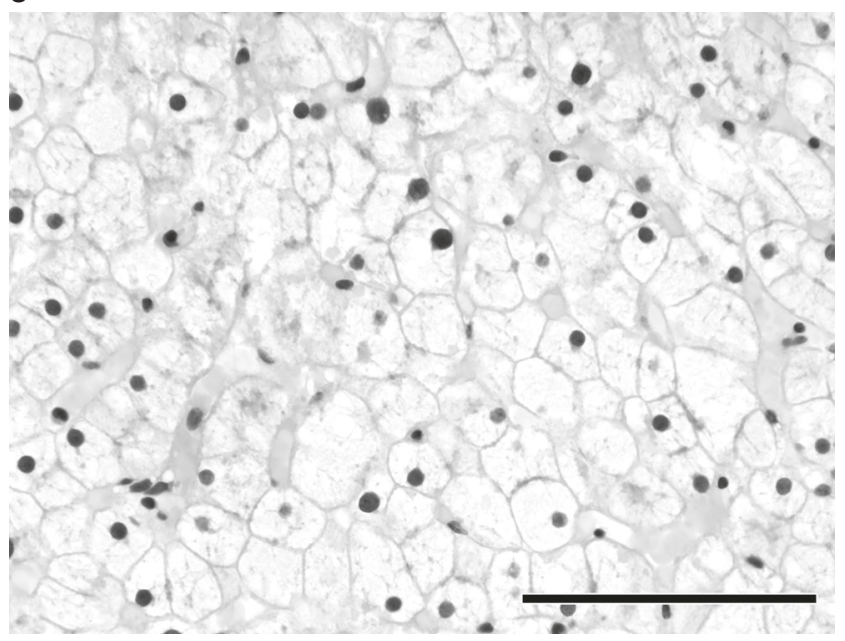

Fig. 1 Typical histologic features of liver in glycogen storage disease (GSD) III. Biopsy obtained from patient 37 at age 9 months. a Low power view of the typical microscopic features seen in a liver biopsy of GSD III, including diffusely light cytoplasm and no apparent fibrosis. b The Masson trichrome stain confirms the lack of fibrosis (no significant blue/green fibrous deposition). c A high power view of hepatocytes shows clear cytoplasm secondary to the accumulation of glycogen, and dark, pyknotic nuclei. Scale bar $=100$ microns

inflammation was rated as minimal to mild in seven patients and moderate in two patients where there was patchy portal or lobular lymphocytic inflammation with occasional interface activity (Fig. 3).

\section{Laboratory findings}

Liver and muscle biochemistry

Age at the time of testing ranged from 2.5 months to 21.6 years (median 9 years). ALT and AST tended to be highest in 
a

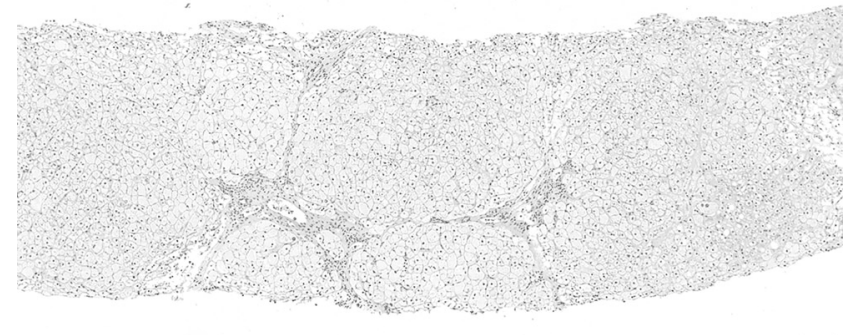

b

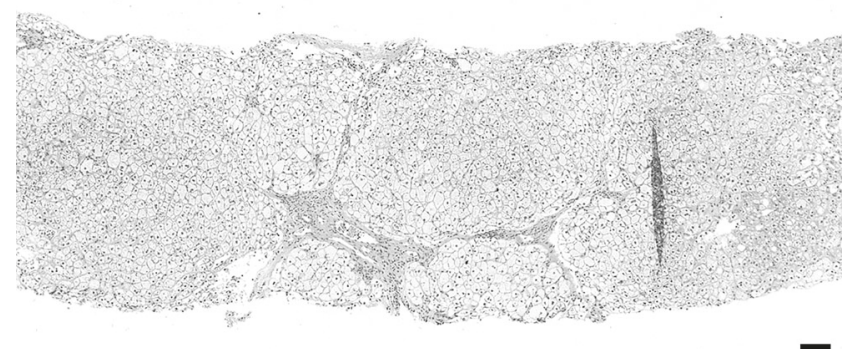

Fig. 2 Advanced fibrosis in glycogen storage disease (GSD) III. Biopsy obtained from patient 35 at age 7 years old. a Low power view of GSD III liver shows dense portal fibrosis with delicate bridges to neighboring portal tracts. $\mathbf{b}$ The Masson trichrome confirms the presence of bridging fibrous as the delicate green bands connect the portal tracts. Scale bar $=100$ microns

the youngest age groups and showed a significant decrease with age. The regression coefficient was $-28(p<0.001)$ and $-29(p<0.001)$ U/L per year, respectively (Fig. 4a, b, respectively) (see Table S2). Values tended to decrease in patients during the teenage years. Median values and ranges were $217.5 \mathrm{U} / \mathrm{L}$ (27 to 1441 ) for ALT and $200 \mathrm{U} / \mathrm{L}$ (25 to 2548), for AST. ALT and AST were significantly correlated $(r=0.932, p<0.0001)$ (Fig. S1.A). GGT was performed in $21 / 26$ patients, and was elevated in seven patients (33\%). Unlike ALT and AST, GGT did not show a significant decrease with age (Fig. 4c); median GGT was $25.5 \mathrm{U} / \mathrm{L}$ (9 to 194, reference range $\leq 19$ ). ${ }^{15}$ Two of 23 patients had mildly elevated PT without a history of bleeding. Platelet counts, bilirubin, albumin, and 3-hydroxybutyrate levels in all patients were within reference limits. Median glucose was $92 \mathrm{mmol} / \mathrm{L}$ (48 to 338). Median CPK was $728 \mathrm{U} / \mathrm{L}$ (38 to 9728). CPK was not significantly correlated with age (regression coefficient $=35, p=0.4$ ) (Fig. 4d), or with ALT and AST ( $r=0.147$ and 0.102 , respectively, $p>0.05$ ) (Fig. S1B, C, respectively).

\section{Lipid profile}

Serum lipids including total cholesterol, triglycerides, and the LDL/HDL ratio tended to be highest in the youngest age groups and showed a significant decrease with age. The regression coefficient was $-2.1 \mathrm{mg} / \mathrm{dL},-18.5 \mathrm{mg} / \mathrm{dL}$, and -0.24 per year, for total cholesterol, triglyceride, and LDL/HDL ratio, respectively $(p<0.001)$ (Fig. $5 \mathbf{a}, \mathbf{b}$ and $\mathbf{e}$, respectively). Median (range) total cholesterol and triglyceride values were $194.5 \mathrm{mg} / \mathrm{dL} \quad(101-421) \quad($ desirable $<200)$ and $214 \mathrm{mg} / \mathrm{dL} \quad(48-1292) \quad($ desirable $<197)$, respectively. Median (range) HDL and LDL, and LDL/HDL values were $28.5 \mathrm{mg} / \mathrm{dL}$ ( 3 to 91$)$ (HDL $<32$ is considered a risk factor for cardiovascular disease), $122.5 \mathrm{mg} / \mathrm{dL}$ (16-279) (LDL borderline high range: $110-129 \mathrm{mg} / \mathrm{dL}$, desirable $<110$ ), and 4.1 (0.2-19.4) (LDL/HDL $<2.5$ considered desirable), respectively.

\section{Urinary glucose tetrasaccharide $\left(G / c_{4}\right)$}

Urinary $\mathrm{Glc}_{4}$ was monitored over 1 to 10 years (median 6.9 years) in all patients except patient 10 (Fig. 4e), and was consistently elevated in a majority. One GSD IIIa patient (patient 15) had normal $\mathrm{Glc}_{4}$ at age 10-13 years, and one GSD IIIb (patient 20) had mild elevations at 7-9 years, and normal values at $12-14$ years. $\mathrm{Glc}_{4}$ was generally more elevated in the youngest patients and tended to decrease with age (regression coefficient was $-0.76 \mathrm{mmol} / \mathrm{mol} \mathrm{CN}$ per year, $p=0.004$ ). This mirrored the trends in the transaminases and serum lipids, and $\mathrm{Glc}_{4}$ was significantly correlated with serum ALT and AST ( $r=0.66$ and 0.72, respectively; $p<0.001$, Fig. 4 f, $\mathbf{g}$, respectively). $\mathrm{Glc}_{4}$ was not significantly correlated with $\mathrm{CPK}$ $\left(r=0.136, p>0.05\right.$, Fig. S1.D). Mean Glc ${ }_{4}$ values for each patient over different age bins are summarized in Table S4.

\section{Imaging studies}

Eighteen hepatic imaging studies from 16 patients were performed at Duke, and included 13 ultrasound (US) and 5 magnetic resonance images (MRI). Two patients had both US and MRI. Median (range) age at the most recent imaging study was 11.5 years (3-21). Findings included hepatomegaly without focal lesions in all 16 patients. Six of 13 patients (46\%) with US had heterogeneous (abnormal) echotexture. No lesions or significant enhancement was noted in all five patients with an MRI. From our observation, 10/16 patients (63\%) had significant enlargement of the left lobe compared with the right lobe (Fig. S2). The remaining 10 patients (10/26) had 9 US and 1 computed tomography (CT) images done at outside hospitals. Three US results showed heterogeneous echotexture, three showed increase echotexture and three showed normal echotexture. All showed hepatomegaly.

\section{Musculoskeletal assessment}

Twenty-one patients had MMT assessments available: 5/21 (24\%) patients had weakness of $\leq 3+$ in 0 to 1 muscle group; $11 / 21$ (52\%) had weakness in 2 to 3 muscle groups; 5/21 (24\%) had weakness in 4 to 6 muscle groups. Scores for each patient are summarized in Table S3. All patients were ambulatory and did not require assistive devices.

\section{Review of literature for GSD III patients}

Several case reports and large cohort studies were reported for GSD III, ${ }^{20-26}$ summarized in Table S1. Large cohorts included patients with ages averaging 26 years, ${ }^{10} 19$ years, ${ }^{12}$ or 17 years. ${ }^{24}$ Liver histology findings were not recorded except for select case reports ${ }^{4,5,11,20,27,28}$ and one cohort study. ${ }^{23}$ It was 

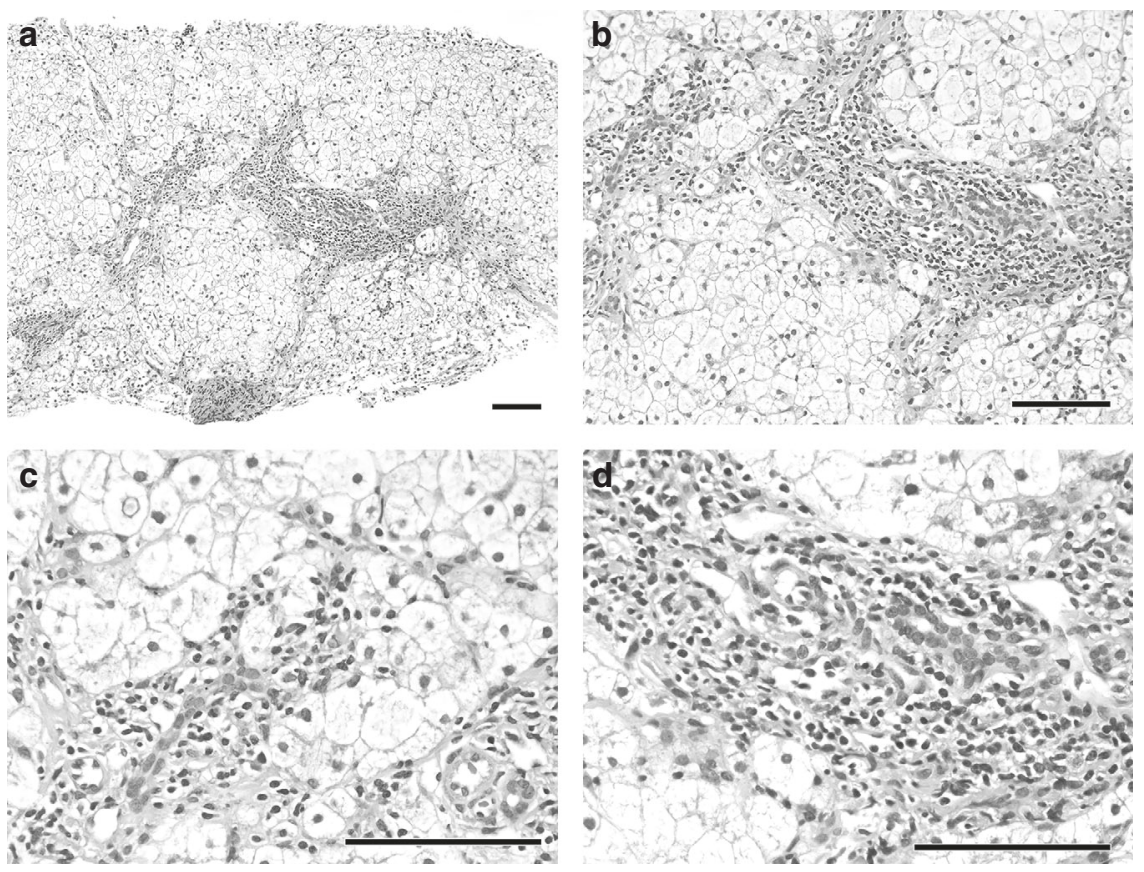

Fig. 3 Glycogen storage disease (GSD) III with increased inflammation. Biopsy obtained from patient 4 at age 2.8 years old. Increased portal-based inflammation observed at low (a) and medium (b) power magnification. Two high power magnification fields (c, d) show dense lymphocytic inflammation with spillover into the lobule, indicating the presence of interface activity. Scale bar $=100$ microns

unanimously described that liver aminotransferases are elevated in the first decade of life and regress thereafter. Long-term hepatic complications have also been reported in two cohort studies with a prevalence of $10-11 \%$ (refs. ${ }^{10,12}$ ). These complications included overt liver findings such as cirrhosis, HCA, and HCC and no histological data were provided in these aforementioned studies.

\section{Review of literature for animal models of GSD III}

Several studies on a canine model and mice models of GSD III have been published. . $7,29,30$ The canine model studies showed liver fibrosis as early as age 4 months, which progressed to cirrhosis at $12-16$ months. ${ }^{30}$ Increasing aminotransferases at 12 months coincided with an increase in liver fibrosis. A decrease in aminotransferases occurred from 16 months and serial biopsies demonstrated progression of hepatic fibrosis leading to cirrhosis. Urinary $\mathrm{Glc}_{4}$ was positively correlated with serum aminotransferases. ${ }^{7}$ Mouse model studies reported similar results with hepatic glycogen accumulation occurring as early as 4 weeks, and with progressive minor fibrosis. ${ }^{6,29}$ Both models showed similar liver biochemical and histological trends as described in GSD III patients.

\section{DISCUSSION}

We investigated the natural course of liver disease in pediatric patients with GSD III. As pediatric patients with GSD III primarily present with hepatic symptoms, this cohort was the focus of our study. In combination with a comprehensive literature review, this study provides insight into hepatic changes in the GSD III pediatric population. Severe liver changes have been described in adult cohorts, but detailed pediatric reports are rare (Table S1). Our study underscores the observation that hepatic fibrosis is a common early finding in children with GSD III, and may be seen as early as the first year of life. Furthermore, the severity of fibrosis (stage 3 or 4 ) observed in $88 \%$ of the children with available liver biopsies, reveals a risk for progression of these hepatic changes to end stage liver disease. The only child without severe fibrosis had stage 2 fibrosis. Our study is also the first to describe a unique histological finding of uncertain significance. We have noted variability in inflammation in the liver biopsies. We speculate that the inflammation may be part of the spectrum of histologic findings in GSD III or may indicate a superimposed liver injury due to an entirely distinct etiology.

In the GSD III cohort described by Sentner et al. ${ }^{1}$ patients with liver complications were identified as those with overt symptoms including HCA, cirrhosis, and HCC. Histological and biochemical details on the early stages and course of the liver disease were lacking in their study, as well as information on dietary therapy adherence and hepatic outcomes. The prevalence of liver complications in their cohort was $11 \%$. However, our study suggests this may be an underestimate, as liver complications that are initially clinically and biochemically silent may not be diagnosed until a later stage in the disease process, as previously described. ${ }^{11,27,28,31}$

Progressive fibrosis and liver cirrhosis associated with decreasing ALT and AST have been observed in the canine model of GSD III. ${ }^{7,30}$ Similar to the canine model, our study shows liver fibrosis can occur at an early age and there is a decrease in aminotransferases with age. We infer that this decrease may be caused by liver disease progression. 

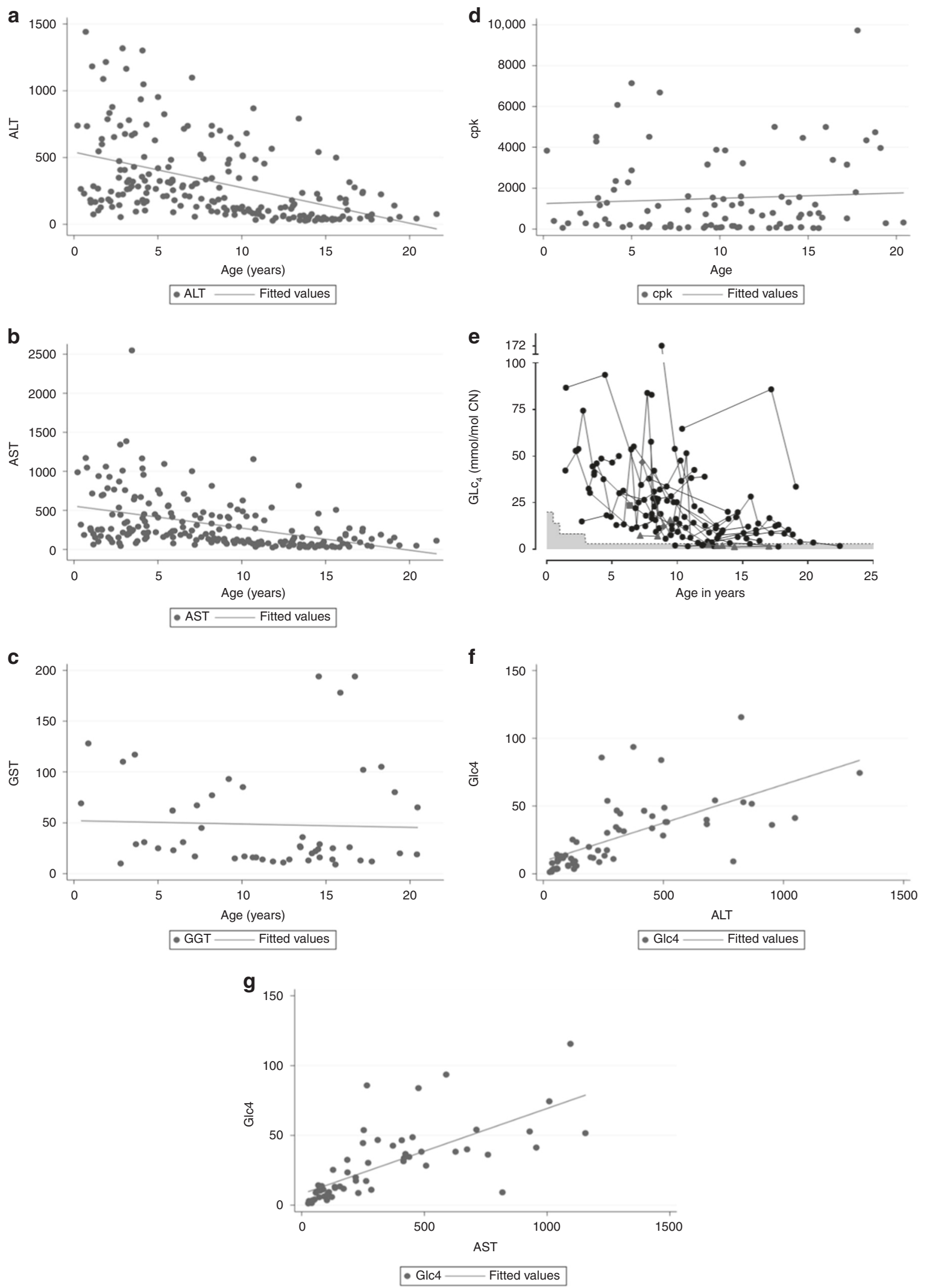
Fig. 4 Correlation of ALT, AST, GGT, CPK, and urine Glc4 levels with age, and of urine Glc4 with ALT and AST in pediatric patients with glycogen storage disease (GSD) III. Correlation of ALT (a), AST (b), GGT (c), CPK (d) (U/L), and urine Glc 4 (e) (mmol/mol creatinine) levels with age (years) in pediatric patients with glycogen storage disease (GSD) III. Correlation of urine $\mathrm{Glc}_{4}$ with ALT (f), and AST (g). Red line represents the regression line. Regression coefficients for AST and ALT with age are -29.5 and -28.5 respectively with $p<0.001$; regression coefficient for GGT is -0.33 with $p=0.9$; and for CPK is 35 with $p=0.4$. Regression coefficients of Glc 4 relative to ALT and AST are 0.66 and 0.72 , respectively, $p<0.001$. e Long-term urinary Glc 4 trends as a function of age (years) in 22 patients with GSD IIla (black symbols) and 3 patients with GSD Illb (blue symbols). Data was not available for patient 10. The dotted line represents the upper limit of age-specific reference ranges. ALT alanine aminotransferase, AST aspartate aminotransferases, CN creatinine, CPK, GGT gamma-glutamyl transferase.

Asymptomatic liver disease progression has been described in other liver diseases such as hepatitis $\mathrm{C}$ virus (HCV) and nonalcoholic fatty liver disease (NAFLD), ${ }^{32,33}$ in which patients may be asymptomatic with normal transaminase levels, despite ongoing fibrosis. It is important to note that while aminotransferases decreased with age in our cohort, it did not normalize for most patients. This may be a result of the muscle disease injury seen in GSD III, in addition to ongoing liver disease.

The trends in urinary $\mathrm{Glc}_{4}$ and its correlation with the transaminases observed in our pediatric cohort were comparable with that observed in the canine model. ${ }^{7}$ In this model, there was a positive correlation between urinary $\mathrm{Glc}_{4}$ and hepatic glycogen $(r=0.751, p=0.008, n=11)$ at 11 to 13 months of age (unpublished data). Furthermore, hepatic glycogen content increased until 12 months of age, but decreased after 16 months with a concomitant increase in fibrosis. ${ }^{30}$ These observations may suggest that in the pediatric GSD III population, $\mathrm{Glc}_{4}$ is derived primarily from hepatic glycogen, and its decrease may be secondary to progression of liver fibrosis. This is supported by the correlation of $\mathrm{Glc}_{4}$ with AST and ALT, but not CPK. However, we cannot exclude the possibility that $\mathrm{Glc}_{4}$ and the transaminases are also derived from muscle; hence, additional tools that are more liver specific are needed for monitoring progression of the liver pathology. Biomarkers such as AST to platelet ratio index ${ }^{34}$ (APRI) and fibrosis 4 $(\text { FIB- } 4)^{35}$ both factor in AST and hence cannot be used in GSD III as results may be impacted by muscle disease. Other biomarkers include fibroblast growth factor 21 (FGF21), which has been studied in other fibrotic liver diseases, ${ }^{36}$ and biotinidase, which has been shown to be elevated in hepatic glycogen storage diseases. ${ }^{37}$ Our findings highlight that while current imaging modalities can detect changes related to overt liver findings such as adenomas and cirrhosis, they have limited ability to detect and monitor fibrosis. Potential noninvasive alternatives include Fibroscan and vibrationcontrolled transient elastography (VCTE), both used for liver fibrosis monitoring in patients with HCV. ${ }^{38,39}$ Longitudinal studies are needed to evaluate these alternative biomarkers and noninvasive tools for monitoring liver disease progression in the GSD III population.

The demonstration of fibrosis in many of our patients suggests that while dietary therapy alleviates symptoms such as hypoglycemia and hepatomegaly, ${ }^{10}$ it may not prevent progression of the liver disease. Animal studies have shown that metabolically controlled GSD III animals develop progressive liver fibrosis. ${ }^{7}$ However, it is unclear whether the same applies to patients with GSD III, and what the role of diet is in preventing liver disease. We recently reported CGM over 7 days in five pediatric patients and one adult with GSD III and showed that glucose levels were within target range $(70-150 \mathrm{mg} / \mathrm{dL}) \quad 94 \%$ of the time. ${ }^{17}$ Home monitoring of blood ketone levels is cost-prohibitive for many of our patients but data on some, when available, showed normal levels with concurrently normal blood glucose levels prior to clinic visits. Based on the above data, CGM data, and adequate growth, our patients may be considered in overall good metabolic control. Long-term studies on glycemic trends are warranted to determine whether the degree of glycemic control is associated with liver injury in GSD III.

Our study is the first comprehensive evaluation of the LDL/ HDL ratio in patients with GSD III. We observed an increase with age in HDL, but no change in LDL resulting in an agerelated decrease in the LDL/HDL ratio. The ratio appeared to normalize with age (Fig. 5e). The LDL/HDL ratio is an excellent predictor of cardiovascular disease (CVD) risk in all populations, and a marker for the effectiveness of lipidlowering treatments. ${ }^{40}$ Further studies are needed to determine whether an increased ratio at younger age significantly increases CVD risk later in life in GSD III.

Our study is also the first to report the median BMI percentile in the GSD III pediatric population. We found that many in our cohort had elevated BMI percentiles (median 80th percentile), possibly a consequence of frequent feeds and the use of uncooked cornstarch, which itself may contribute to processes such as NAFLD.

Our study has limitations. First, because it is retrospective, it is limited by the data available at certain time points. Second, the histological data is cross-sectional as biopsies are not routinely performed as the standard of care. Thus, the selection process may have been biased and this limits our description of histological liver disease progression. The strength of this study relies on a patient cohort carefully managed at a single center by a team with expertise in GSD III, including a metabolic dietitian to ensure metabolic control. Furthermore, we acknowledge that caution needs to be taken when extrapolating data from animal models to human diseases. Lastly, we could draw no conclusions on whether there was a correlation of liver disease 

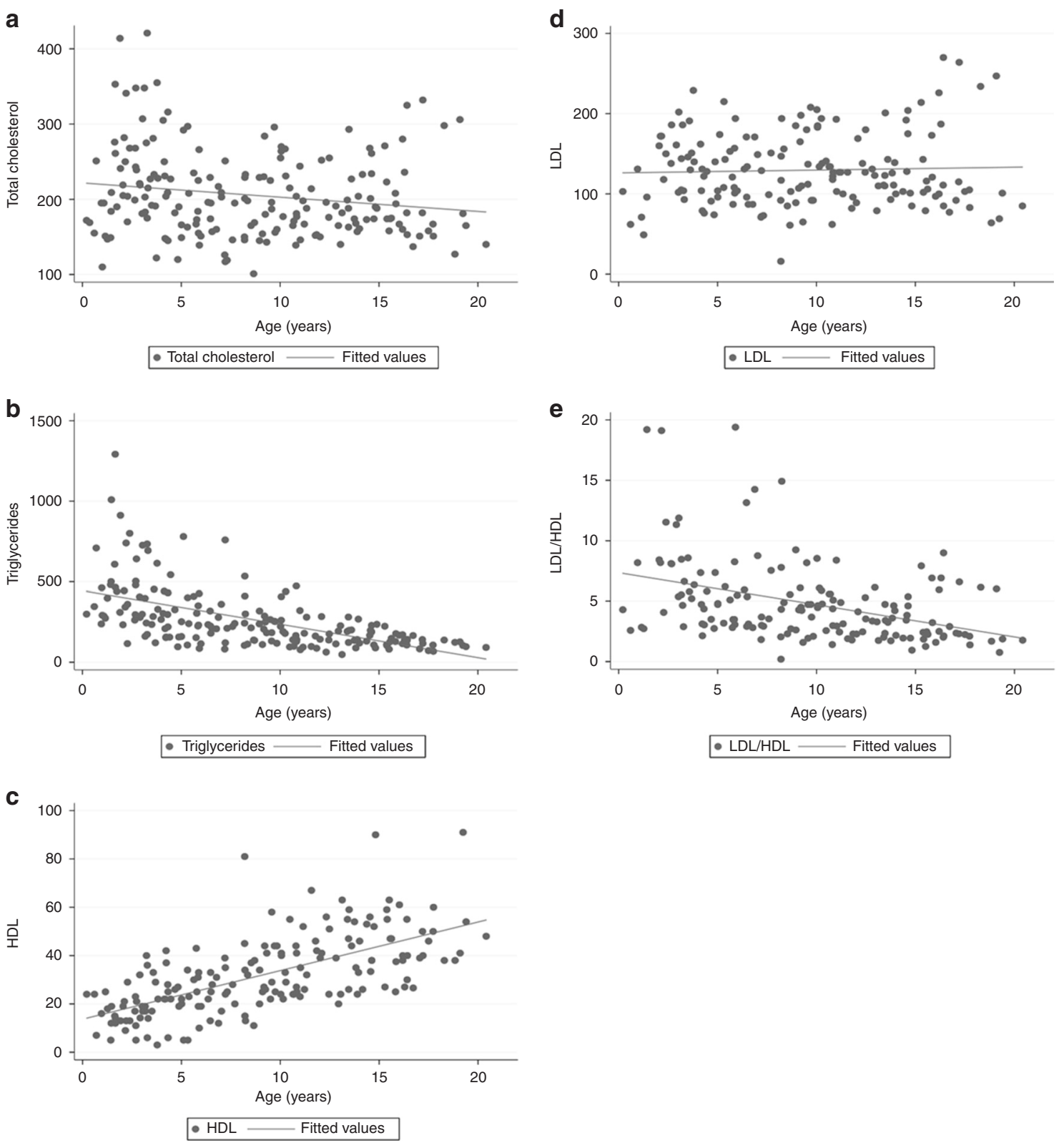

Fig. 5 Correlation of serum lipid levels with age in pediatric patients with glycogen storage disease (GSD) III. Correlation of serum lipid levels with age in pediatric patients with glycogen storage disease (GSD) III, including total cholesterol (a), triglyceride (b), HDL (c), LDL (d) (all in mg/dL), and LDL/HDL (e). Red line represents the regression line. Regression coefficients for all are: $-2.1,-18.5,1.5,-0.3$, and -0.2 respectively, $p<0.001$. HDL high density lipoprotein, $L D L$ low density lipoprotein.

severity with genotype, as there were few recurrent pathogenic variants, and histological data was not available for the entire cohort. Prospective studies on international GSD III patient registries are needed to better understand the impact of genotype on disease severity.

In conclusion, as life expectancy of individuals with GSD III improves, there is a risk that clinically silent liver fibrosis will progress and result in severe hepatic complications later in life. The pathogenic mechanism of liver disease in GSD III is currently unknown. Current biomarkers such as transaminases and urinary $\mathrm{Glc}_{4}$ may be useful for monitoring liver disease in patients yet may be affected by muscle disease. Therefore, more specific biomarkers and imaging tools are needed to accurately monitor the liver disease. We have shown that it is important to closely monitor patients over time for liver manifestations, despite the normalization of liver enzymes, as fibrosis may be prevalent within the pediatric GSD III population with risk for progression. Liver fibrosis is often noted early in the disease course, and can occur as early as the first year of life. Future therapeutic strategies should aim for an early intervention in GSD III, prior to progression of hepatic fibrosis. 


\section{SUPPLEMENTARY INFORMATION}

The online version of this article (https://doi.org/10.1038/s41436019-0561-7) contains supplementary material, which is available to authorized users.

\section{ACKNOWLEDGEMENTS}

We thank the patients with GSD III who have dedicated their time and effort to help us better understand GSD III. We thank Elizabeth Brooks and Baodong Sun of Duke University for sharing unpublished animal study results. A portion of this study was funded by Valerion Therapeutics.

\section{DISCLOSURE}

The authors declare no conflicts of interest.

Publisher's note: Springer Nature remains neutral with regard to jurisdictional claims in published maps and institutional affiliations.

\section{REFERENCES}

1. Van Hoof $F$, Hers HG. The subgroups of type 3 glycogenosis. Eur J Biochem. 1967;2:265-270.

2. Endo $Y$, Horinishi A, Vorgerd $M$ et al. Molecular analysis of the AGL gene: heterogeneity of mutations in patients with glycogen storage disease type III from Germany, Canada, Afghanistan, Iran, and Turkey. J Hum Genet. 2006;51:958-963.

3. Goldstein JL, Austin SL, Boyette $\mathrm{K}$ et al. Molecular analysis of the $\mathrm{AGL}$ gene: identification of 25 novel mutations and evidence of genetic heterogeneity in patients with glycogen storage disease type III. Genet Med. 2010;12:424-430.

4. Haagsma EB, Smit GP, Niezen-Koning KE et al. Type Illb glycogen storage disease associated with end-stage cirrhosis and hepatocellular carcinoma. The Liver Transplant Group. Hepatology. 1997;25:537-540.

5. Siciliano M, De Candia E, Ballarin $S$ et al. Hepatocellular carcinoma complicating liver cirrhosis in type Illa glycogen storage disease. J Clin Gastroenterol. 2000;31:80-82.

6. Liu KM, Wu JY, Chen YT. Mouse model of glycogen storage disease type III. Mol Genet Metab. 2014;111:467-476.

7. Brooks ED, Yi H, Austin SL et al. Natural progression of canine glycogen storage disease type Illa. Comp Med. 2016;66:41-51.

8. Kumlien J, Chester MA, Lindberg BS et al. Urinary excretion of a glucosecontaining tetrasaccharide. A parameter for increased degradation of glycogen. Clin Chim Acta. 1988;176:39-48.

9. Kishnani PS, Austin SL, Arn P et al. Glycogen storage disease type III diagnosis and management guidelines. Genet Med. 2010;12:446-463.

10. Hershkovitz E, Forschner I, Mandel $\mathrm{H}$ et al. Glycogen storage disease type III in Israel: presentation and long-term outcome. Pediatr Endocrinol Rev. 2014;11:318-323.

11. Demo E, Frush D, Gottfried $M$ et al. Glycogen storage disease type IIIhepatocellular carcinoma a long-term complication? J Hepatol. 2007:46:492-498.

12. Sentner CP, Hoogeveen IJ, Weinstein DA et al. Glycogen storage disease type III: diagnosis, genotype, management, clinical course and outcome. J Inherit Metab Dis. 2016;39:697-704.

13. Richards S, Aziz N, Bale S.ACMG Laboratory Quality Assurance Committee et al. Standards and guidelines for the interpretation of sequence variants: a joint consensus recommendation of the American College of Medical Genetics and Genomics and the Association for Molecular Pathology. Genet Med. 2015;17:405-424.

14. Austin SL, Proia AD, Spencer-Manzon MJ et al. Cardiac pathology in glycogen storage disease type III. JIMD Rep. 2012;6:65-72.

15. Colantonio DA, Kyriakopoulou L, Chan MK et al. Closing the gaps in pediatric laboratory reference intervals: a CALIPER database of 40 biochemical markers in a healthy and multiethnic population of children. Clin Chem. 2012;58:854-868.
16. Young SP, Stevens RD, An Y et al. Analysis of a glucose tetrasaccharide elevated in Pompe disease by stable isotope dilution-electrospray ionization tandem mass spectrometry. Anal Biochem. 2003;316:175-180.

17. Herbert $M$, Pendyal $S$, Rairikar $M$ et al. Role of continuous glucose monitoring in the management of glycogen storage disorders. J Inherit Metab Dis. 2018;41:917-927.

18. Council MR. Aids to Examination of the Peripheral Nervous System. London: Her Majesty's Stationary Office 1976, Memorandum No. 45.

19. Batts KP, Ludwig J. Chronic hepatitis. An update on terminology and reporting. Am J Surg Pathol. 1995;19:1409-1417.

20. Okuda S, Kanda F, Takahashi K et al. Fatal liver cirrhosis and esophageal variceal hemorrhage in a patient with type Illa glycogen storage disease. Intern Med. 1998;37:1055-1057.

21. Karwowski C, Galambos C, Finegold D et al. Markedly elevated serum transaminases in glycogen storage disease type III. J Pediatr Gastroenterol Nutr. 2011;52:621-623.

22. Quackenbush D, Devito J, Garibaldi L et al. Late presentation of glycogen storage disease types la and III in children with short stature and hepatomegaly. J Pediatr Endocrinol Metab. 2018;31:473-478.

23. Coleman RA, Winter HS, Wolf B et al. Glycogen storage disease type III (glycogen debranching enzyme deficiency): correlation of biochemical defects with myopathy and cardiomyopathy. Ann Intern Med. 1992;116:896-900.

24. Labrune P, Trioche P, Duvaltier I et al. Hepatocellular adenomas in glycogen storage disease type I and III: a series of 43 patients and review of the literature. J Pediatr Gastroenterol Nutr. 1997;24:276-279.

25. Bhuiyan J, Al Odaib AN, Ozand PT. A simple, rapid test for the differential diagnosis of glycogen storage disease type 3. Clin Chim Acta. 2003;335:21-26.

26. Lucchiari S, Santoro D, Pagliarani S et al. Clinical, biochemical and genetic features of glycogen debranching enzyme deficiency. Acta Myol. 2007:26:72-74.

27. Fellows IW, Lowe JS, Ogilvie AL et al. Type III glycogenosis presenting as liver disease in adults with atypical histological features. J Clin Pathol. 1983;36:431-434

28. Oterdoom $L H$, Verweij $K E$, Biermann $K$ et al. Hepatocellular adenomas and carcinoma in asymptomatic, non-cirrhotic type III glycogen storage disease. J Gastrointestin Liver Dis. 2015;24:515-518.

29. Pagliarani S, Lucchiari S, Ulzi G et al. Glycogen storage disease type III: a novel Agl knockout mouse model. Biochim Biophys Acta. 2014; 1842:2318-2328

30. Yi H, Thurberg BL, Curtis $\mathrm{S}$ et al. Characterization of a canine model of glycogen storage disease type Illa. Dis Model Mech. 2012;5:804-811.

31. Matern D, Starzl TE, Arnaout $W$ et al. Liver transplantation for glycogen storage disease types I, III, and IV. Eur J Pediatr. 1999;158 suppl 2:S43-8.

32. Mofrad $\mathrm{P}$, Contos MJ, Haque M et al. Clinical and histologic spectrum of nonalcoholic fatty liver disease associated with normal ALT values. Hepatology. 2003;37:1286-1292.

33. Alberti $A$, Morsica $G$, Chemello $L$ et al. Hepatitis $C$ viraemia and liver disease in symptom-free individuals with anti-HCV. Lancet. 1992;340:697-698.

34. Wai CT, Greenson JK, Fontana RJ et al. A simple noninvasive index can predict both significant fibrosis and cirrhosis in patients with chronic hepatitis C. Hepatology. 2003;38:518-526.

35. Sterling RK, Lissen E, Clumeck N.APRICOT Clinical Investigators. et al. Development of a simple noninvasive index to predict significant fibrosis in patients with HIV/HCV coinfection. Hepatology. 2006;43:1317-1325.

36. Krautbauer S, Rein-Fischboeck L, Haberl EM et al. Circulating fibroblast growth factor 21 in patients with liver cirrhosis. Clin Exp Med. 2018;18:63-69.

37. Paesold-Burda $P$, Baumgartner $M R$, Santer $R$ et al. Elevated serum biotinidase activity in hepatic glycogen storage disorders-a convenient biomarker. J Inherit Metab Dis. 2007;30:896-902.

38. Paranagua-Vezozzo DC, Andrade A, Mazo DF et al. Concordance of noninvasive mechanical and serum tests for liver fibrosis evaluation in chronic hepatitis C. World J Hepatol. 2017;9:436-442.

39. Singh $S$, Loomba R. Role of two-dimensional shear wave elastography in the assessment of chronic liver diseases. Hepatology. 2018;67:13-15.

40. Manninen $V$, Tenkanen $L$, Koskinen $P$ et al. Joint effects of serum triglyceride and LDL cholesterol and HDL cholesterol concentrations on coronary heart disease risk in the Helsinki Heart Study. Implications for treatment. Circulation. 1992;85:37-45. 